\title{
Horacio J. A. Rimoldi y la psicología científica en Latinoamérica d
}

\author{
María Andrea Piñeda a \& b 1요, \& Jorge Chavez ${ }^{\mathfrak{C} \mathbb{C}^{2}}$
}

Consejo Nacional de Investigaciones Científicas y Técnicas, San Luis, Argentina a ; Universidad Nacional de San Luis, San Luis, Argentina b; Universidad de la República, Montevideo, Uruguay ${ }^{\mathrm{c}}$

\section{RESUMEN}

Enfocado en la figura del Dr. Horacio Rimoldi, miembro destacado de la Sociedad Interamericana de Psicología, este estudio histórico describe la sistemática circulación de conocimientos y de prácticas de formación que él propició entre universidades de Argentina, Estados Unidos, Puerto Rico y Uruguay entre principios de 1950 y 1970. Dichas acciones tuvieron el propósito de fortalecer el desarrollo de la ciencia psicológica en Latinoamérica cuando allí se producía la profesionalización disciplinar. Se recuperó un voluminoso corpus de fuentes inéditas posibilitando analizar más de 160 documentos institucionales y cartas entre académicos. Se describen los cursos dictados por Rimoldi y sus intentos de organizar un laboratorio en la Universidad de la República de Uruguay, entre 1951 y 1952. También, entre 1957 y 1969 , se registran las colaboraciones entre Universidad Nacional de Córdoba, Universidad de Buenos Aires, CONICET, Universidad de Puerto Rico, Sociedad de Psicología de Puerto Rico y Universidad de Loyola. Con resultados disímiles, según los complejos contextos locales y los avatares institucionales, se reflejan procesos de profesionalización y de constitución de centros académicos orientados al desarrollo de una psicología como ciencia básica, autónoma, próxima a las ciencias naturales, que procura una formación universitaria centrada en la investigación y abierta a la agenda internacional.

Palabras Clave

historia de la psicología; formación en psicología; universidades; psicometría; América Latina

\begin{abstract}
Focusing on Dr. Horacio Rimoldi, a prominent member of the Interamerican Society of Psychology, this historical study describes the regular circulation of knowledge and training practices that he promoted between universities in Uruguay, the United States, Argentina and Puerto Rico between the early 1950s and 1970. The purpose of these actions was to strengthen the development of psychological science in Latin America at a time when the professionalisation of the discipline was taking place there. A considerable corpus of unpublished sources was recovered, making it possible to analyse more than 160 institutional documents and letters between academics. It describes the courses taught by Rimoldi and his attempts to organise a laboratory at the Universidad de la República de Uruguay, between 1951 and 1952. Also, between 1957 and 1969, collaborations with Universidad Nacional de Córdoba, Universidad de Buenos Aires, CONICET, Universidad de Puerto Rico, Sociedad de Psicología de Puerto Rico and Universidad de Loyola are documented. Dissimilar results were achieved, depending on the complex local contexts and institutional vicissitudes. The interactions highlight processes of professionalization and the constitution of academic centres oriented towards the development of psychology as a basic, autonomous science, close to the natural sciences, which seeks university education focused on research and open to the international agenda.
\end{abstract}

Keyswords

history of psychology; psychology education; universities; psychometry; Latin America

\footnotetext{
1 Correspondence about this article should be addressed María Andrea Piñeda: mapineda@unsl.edu.ar

2 Conflicts of Interest: The authors declare that the research was conducted in the absence of any commercial or financial relationships that could be construed as a potential conflict of interest.
} 
Horacio Rimoldi J. A. and Scientific Psychology in Latinamerica

\section{Introducción}

En el presente trabajo se busca destacar la sistemática circulación de conocimientos y de prácticas de formación en psicología entre universidades de Argentina, Estados Unidos, Puerto Rico y Uruguay, que fueron propiciadas por Horacio J. A. Rimoldi (1913-2006) entre principios de 1950 y 1970. Dichas acciones tuvieron el propósito de constituir centros de desarrollo de ciencia psicológica en Latinoamérica cuando allí se producía el proceso de profesionalización disciplinar.

En este número especial dedicado a celebrar la rica historia de la Sociedad Interamericana de Psicología (SIP), ponemos de relieve la figura del académico argentino Horacio Rimoldi, quien fue miembro de esta sociedad ejerciendo como editor de la Revista Interamericana de Psicología (RIP) entre 1975 y 1976, y cuya destacada trayectoria recorre y vincula las Américas. Algunas historias locales reconstruyeron su temprana labor (Calabresi, 2010) y su papel en la gestión de políticas científicas en Argentina (Piñeda, 2020). Otras contribuciones analizaron su significación para la psicología Ibero y latinoamericana (Carpintero, 2006, Revista Latinoamericana de Psicología, 1986). Así, conocemos que tras graduarse con honores como médico de la Universidad de Buenos Aires (1938), su maestro Bernardo Houssay (1887-1971), premio Nobel en Medicina y Fisiología (1947) lo orientó para formarse en psicometría en Inglaterra. Entre 1939 y 1941 abordó estudios sobre la fatiga y se entrenó en métodos factoriales en la Universidad de Oxford bajo la dirección de William Stephenson (19021989), discípulo directo de Charles Spearman (1863-1945). Poco antes de finalizar su doctorado, fue recomendado por Houssay para desempeñarse como Profesor de Biología y de Psicología Experimental y para organizar un Laboratorio de Psicología Experimental en la flamante Universidad Nacional de Cuyo (Mendoza, Argentina), por lo que se le solicitó regresar. No exento de dificultades presupuestarias para montar la infraestructura necesaria, Rimoldi organizó el laboratorio que le fue encomendado. Allí entró en contacto con Emilio Mira y López (1896-1964) y, por medio de él, con su discípula Nuria Cortada (1921-2013) con la que logró la primera estandarización extranjera de las matrices progresivas de Raven. Tras sucesos políticos que ocasionaron el desplazamiento fuera de las universidades públicas de académicos cercanos a Houssay que eran percibidos como adversarios del gobierno peronista, en 1946 Rimoldi decidió trasladarse a Estados Unidos. En 1948 obtuvo el título de Doctor en Psicología por la Universidad de Chicago 
bajo la dirección de Louis Thurstone (1887-1955) en cuyo Laboratorio de Psicometría se entrenó en el estudio de los procesos cognitivos y tácticas empleadas en la resolución de problemas. Permaneció en esa universidad hasta 1951. En ese período también fue Profesor Invitado de Psicometría en el Instituto de Psicología Aplicada de la Universidad Nacional de Colombia (1949) a instancias de la Profesora Mercedes Rodrigo (18911982), psicóloga española que fundó la primera carrera de psicología de ese país. Entre enero de 1951 y fines de 1952, la Facultad de Humanidades y Ciencias de la Universidad de la República (Uruguay) lo contrató para organizar la carrera de psicología. A continuación, regresó a Estados Unidos para incorporarse al Educational Testing Service de Princeton. Entre 1955 y 1970, Rimoldi se desempeñó como Profesor de la Universidad de Loyola donde fundó y dirigió el Laboratorio de Psicometría. Desde allí, se convirtió en referencia internacional dirigiendo más de 40 tesis y estancias de investigadores de diversos países. Tras dicha etapa, regresó definitivamente a la Argentina, donde se incorporó con el máximo rango de Investigador al Consejo Nacional de Investigaciones Científicas y Técnicas (CONICET), dirigiendo el Centro Interdisciplinario de Investigaciones en Psicología Matemática y Experimental (CIIPME). Houssay y Thurstone fueron sus mayores influencias (Oiberman, 2003) y a su abrigo forjó su concepción de psicología como ciencia básica, autónoma, cercana a las ciencias naturales, para cuyo desarrollo hacía falta una formación universitaria centrada en la investigación y abierta a la agenda internacional. A Rimoldi no se le escapaban las condiciones que las políticas académicas debían asegurar: financiamiento para la organización de institutos y laboratorios, cargos docentes de tiempo completo, contratación de profesores visitantes extranjeros y becas para la formación de posgrado en el exterior de jóvenes bien elegidos a los que se les aseguren las condiciones adecuadas para regresar al país (Piñeda, 2020).

Pero esas no eran las circunstancias más habituales en las universidades latinoamericanas. En el período posterior a la segunda guerra mundial, en alternancia de gobiernos autoritarios y populistas, América Latina asistió a un proceso de industrialización acompañado de aumento de población de centros urbanos y mejora del nivel general de educación. Desde una concepción desarrollista emanada de la Comisión Económica para América Latina (CEPAL), se sostenía la noción de que las ciencias y las universidades jugarían un papel central en el desarrollo socioeconómico. Desde la década de 1960 se gestaron políticas públicas modernizadoras de las universidades otorgando un papel central a la investigación. Se organizaron institutos, programas de formación de posgrado y se fomentaron cargos docentes universitarios con dedicación exclusiva. Pero, 
al mismo tiempo que se masificó la matrícula, los presupuestos resultaron insuficientes para atender las demandas. Se desfinanció y deterioró la investigación universitaria, en consecuencia, los Consejos de Ciencia y Tecnología pasaron a financiar y a definir los criterios en política científica (Vessuri, 1994).

Por su parte, la formación universitaria en psicología en América Latina no fue ajena a las condiciones precedentes. Se ha documentado que los primeros programas de grado se iniciaron entre 1947 y 1974 en 21 países (Klappenbach, Vázquez-Ferrero \& Gallegos, 2018). Se ha analizado que la Conferencia de Boulder sobre el entrenamiento en psicología clínica (1949) inspiró los programas de formación de la región, y sus lineamientos aún seguían vigentes en los debates de 1974 sobre el modelo latinoamericano de formación científica y profesional que acontecieron en Bogotá durante la primera Conferencia Latinoamericana para el Entrenamiento en psicología (Gallegos, 2010). En Argentina, entre 1950 y 1953 existían tres carreras de perfil psicotécnico que antecedieron a la organización de la primera carrera de Psicólogo en la Facultad de Filosofía y Letras de la Universidad Nacional del Litoral en 1954. En la primera década, este programa se organizó en otras 13 universidades. Bajo la denominación de Psicólogo o de Licenciado en Psicología, el título de grado habilitaba al ejercicio de la profesión liberal cuyo perfil fue cambiando desde 1960. El modelo psicotécnico fue desplazado por un enfoque predominantemente clínico y psicoanalítico, que puso en crisis la hegemonía médica sobre ese campo profesional (Klappenbach, 2018). La autonomía respecto del médico en el ejercicio de la profesión de psicólogo en el área clínica recién fue legalizada a mediados de 1980. La disputaban tanto los médicos psicoanalistas que abundaban en los planteles docentes de psicología contribuyendo al sesgo, como aquellos críticos de la cientificidad de ese perfil que se encontraban más próximos a la Facultad de Medicina de la UBA y al CONICET (Klappenbach, 2012). En Uruguay, en la Universidad de la República, el Curso de Psicología Aplicada a la Infancia comenzó en 1950 en la la Sección Auxiliares del Médico de la Facultad de Medicina, otorgando el título de Técnico en Psicología Infantil (Asociación de Psicólogos Universitarios Egresados de la Escuela de Tecnología Médica, 1983). En 1956, en la Facultad de Humanidades y Ciencias, se creó la Licenciatura en Psicología. Esto ocurrió después de un controvertido proceso que dejó expuestas las tensiones que emergieron en torno al control de la formación en psicología que rápidamente se trasladó al ejercicio profesional. La lucha por el control del ejercicio de la psicología y especialmente sobre la práctica de la psicoterapia, enfrentó a psiquiatras y psicólogos desde que se inició la 
carrera de la FHC en 1956 hasta la promulgación de la ley del ejercicio profesional de la psicología en 1999 (Chavez \& Martinez Sena, 2021). En Puerto Rico, se siguió un camino diferente. Por su proximidad a Estados Unidos y sus relaciones diplomáticas como estado libre y asociado, al igual que el país del norte, el título de posgrado era indispensable para habilitar la práctica profesional. Por lo que en la década de 1930 ya se contaba con especializaciones en psicología para profesionales de los campos de las ciencias sociales y educación, y también se hizo fluida la circulación de académicos que realizaron estudios doctorales en Estados Unidos, lográndose un nivel de profesionalización que ameritaba la creación de la Sociedad de Psicología de Puerto Rico en 1954. Se organizaron los primeros programas de Maestría en Psicología en 1966 en la Universidad de Puerto Rico y en 1967 en el Instituto Psicológico de Puerto Rico que luego fue renombrado Universidad Carlos Albizu (Ortiz-Torres, 2013, Rivera \& Maldonado, 2000, Roca de Torres, 1999).

Carl F. Hereford, académico de la Universidad de Texas y primer editor de la RIP (1967-1970), durante 1965 visitó y analizó las condiciones de 32 programas universitarios de psicología latinoamericanos. En su reporte (Hereford, 1966) describió tres obstáculos comunes para producir investigación científica. Primero, la escasez presupuestaria para organizar institutos con infraestructura adecuada, disponer de bibliotecas y laboratorios actualizados, y pagar salarios a los docentes, que no contaban con cargos de dedicación suficiente para tareas investigativas ni con formación especializada en ciertas áreas. En efecto, las carreras de psicología fueron organizadas en facultades de humanidades, filosofía, educación y medicina, y los docentes estaban formados en esas disciplinas. La escasez de psicólogos cualificados en áreas específicas y la falta de instalaciones suficientes para las masivas matrículas, dejaban a los estudiantes sin oportunidades de realizar prácticas supervisadas. Por otro lado, Hereford señalaba que la propia cultura latina operaba como un obstáculo, inclinándose por una psicología cercana a las humanidades y a las letras, porque modelaba carreras de psicología con perfil teórico, propensas a la recepción de la clínica francesa y del psicoanálisis, pero resistentes a la psicología experimental. Al mismo tiempo, el reporte celebraba el creciente número de académicos latinos que buscaban alternativas de formación en universidades estadounidenses, gracias a becas cada vez más fáciles de obtener.

Desde una perspectiva policéntrica (Brock, 2014, Danziger, 1996), pretendemos analizar la sistemática circulación de conocimiento y de prácticas de formación que Rimoldi vehiculizó entre principios de 1950 y 1970 en Uruguay, Argentina y Puerto Rico, 
aún cuando estaba radicado en Estados Unidos, con el propósito de constituir centros de investigación orientados hacia una psicología próxima a las ciencias naturales, en contraste con los modelos más cercanos a las humanidades y a las letras que florecían en Latinoamérica. Una mirada desde la sociología de las profesiones (Abbott, 1988; Freidson, 1996), también nos servirá para comprender las dinámicas y tensiones que la introducción de estas prácticas evidenciaron en momentos donde se definía la profesionalización de la psicología, su enseñanza, la construcción de la identidad profesional y el control del mercado en torno al ejercicio profesional.

\section{Método}

Siguiendo una metodología histórica de análisis documental (Benjamin, 2006, Klappenbach, 2014, Jacó-Vilela, Espírito-Santo, Degani-Carneiro, Goes, \& Vasconcellos, 2016), se realizó el relevamiento, lectura y análisis de contenido de un voluminoso corpus de fuentes inéditas.

\section{Diseño y materiales}

Por un lado, se realizó un relevamiento en el Archivo Mario Berta ubicado en el Archivo de Historia de la Medicina de la Facultad de Medicina de la UdelaR, donde se recuperaron 30 documentos referidos al período en el que se desarrollan las acciones de Rimoldi dirigidas a instalar una carrera de formación en psicología (1950-1953). Los documentos seleccionados fueron informes de actividades de Horacio Rimoldi y Mario Berta sobre sus respectivos cursos de psicología, comunicaciones personales de ambos profesores dirigidas a autoridades de la Facultad de Humanidades y Ciencias y resoluciones de la Facultad referidas a los cursos de psicología de Rimoldi y Berta. Por otra parte, se recuperaron y digitalizaron 133 cartas escritas entre 1951 y 1969. Entre 1951 y 1955, fueron escritas 5 de ellas con su maestro Louis Thurstone (1887-1955) y relatan los proyectos de Rimoldi de organizar un laboratorio de Psicología en Uruguay, sus desventuras en el intento y sus planes de regresar a Estados Unidos. Entre 1955 y 1969, se registran dos grupos de cartas. El primero está constituido por 102 documentos intercambiados con académicos argentinos (1957-1969): a) Universidad Nacional de Córdoba (autoridades: Raúl Piérola, Adelmo Montenegro, Tomás Fulgueira, Jorge Orgaz, Rogelio Nores Martínez; estudiantes: Rosalía Amitrano de Paiva y Enrique Saforcada). b) UBA (Nuria Cortada, su esposo Manuel Kohan y Jorge Insúa). c) CONICET 
(directivos: Houssay, Prieto, Tramezzani y Cámola; becarios: A. M. Insúa y Pecci Saavedra; investigadores: Antonio Battro e Ives Lys Danna). El segundo, lo componen 26 cartas (1958-1965) con académicos de la Asociación de Psicología y de la Universidad de Puerto Rico (Mercedes Rodrigo, Juan Rossello, Jorge Morales Yordán, R.G. Cundiff, Michael Cooney, Raúl B. Díaz Cataldo, Jaime Benítez, Adriana Guzmán, Juan Nicolás Martínez y Álvarez). Las cartas están en posesión de la biblioteca del CIIPME, sin catalogar, en carpetas que caratuladas según el material archivado (ej. inicial del apellido, ciudad de procedencia o filiación institucional de las personas con las que mantiene correspondencia). Por esa razón, solo se puede indicar una ubicación aproximada en el fichero.

\section{Procedimiento y análisis de datos}

El material, en su totalidad, fue registrado en una base de datos y fue leído de manera diacrónica y sincrónica, se elaboraron resúmenes del contenido y se copiaron citas textuales de interés. El análisis de contenido se centró en la categoría interacciones entre los actores institucionales y se consideraron las siguientes sub-categorías: invitaciones, solicitudes, financiamientos, acceso a publicaciones, dictado de cursos, asesoramientos, recomendaciones profesionales, concursos y evaluaciones de desempeño, recepción de estudiantes de posgrado en la Universidad de Loyola. Una síntesis de esos hallazgos se presenta en dos secciones. En la primera, se describen las interacciones que tuvieron lugar en Uruguay, entre 1951 y 1952. En la segunda parte, se relatan los intercambios ocurridos entre 1957 y 1969 entre Rimoldi -radicado en Estados Unidos- y los académicos de Argentina y Puerto Rico.

\section{Resultados}

\section{Horacio Rimoldi y su proyecto de psicología científica para Uruguay}

El vínculo de Rimoldi con la psicología en el Uruguay se inició en el marco de la investigación sobre El normotipo del niño y el adolescente uruguayo desarrollada entre abril de 1944 y septiembre de 1945. Los organismos de Educación Primaria y Secundaria contrataron a Emilio Mira y López como Director de la investigación que contó con la labor del Laboratorio de Psicopedagogía Sebastián Morey Otero integrado por maestras especializadas en técnicas psicométricas. Horacio Rimoldi fue invitado por Mira y López a dictar un curso sobre Estadística Psicopedagógica y a elaborar tres escalas de 
inteligencia (verbal, espacial y abstracta), que fueron estandarizadas y baremadas, proveyendo al Laboratorio de una batería para la medida de la inteligencia de los adolescentes (Carbonell de Grompone, 1947).

A partir de esta experiencia, Rimoldi continuó en contacto con el Laboratorio de Psicopedagogía y especialmente con un grupo de alumnas y colegas con las que volverá a encontrarse en 1951, en ocasión que el decano de la Facultad de Humanidades y Ciencias (FHC) de la Universidad de la República (Uruguay), el Dr. Justino Jiménez de Aréchaga, lo convocó para elaborar una propuesta de creación de un Instituto de Psicología y organizar la Licenciatura en Psicología en dicha institución.

El 13 de noviembre de 1950, Jiménez de Aréchaga escribió a Horacio Rimoldi, quien se encontraba en Chicago, invitándolo a Uruguay con el objetivo de "proceder, dentro de la Facultad, a la instalación y organización de varios cursos de psicología que pudieran, una vez estructurados en forma conveniente, servir también para la expedición de un grado de licenciado en esta actividad" (Rimoldi, 1951a). Rimoldi aceptó, contando con la posibilidad de organizar de manera sistemática la enseñanza y la investigación en psicología. Para alcanzar esa meta, el 24 de noviembre de 1950 Rimoldi consultó a Jiménez de Aréchaga sobre el presupuesto anual del que dispondría, y agregaba una estimación de lo que consideraba necesario para iniciar sus actividades. Su solicitud incluía suscripciones a revistas, la compra de libros para iniciar una biblioteca moderna, pruebas mentales, una máquina de calcular, un grabador de alambre para grabar conferencias, un proyector y un monto inicial de entre 1000 y 1500 U\$ para la compra inicial de aparatos.

Las condiciones planteadas por Rimoldi no llegaron a concretarse mínimamente, del presupuesto solicitado se otorgaron 500 U\$, tampoco se dispuso de la mayoría de los materiales y publicaciones solicitados, lo que refleja desde un inicio las dificultades a las que se enfrentó para concretar sus objetivos. A esto, se suma la compleja situación institucional de la FHC al momento de su arribo y los contradictorios mensajes en torno al interés de la institución universitaria para crear la Licenciatura en Psicología.

El mismo año en que el Decano de FHC invitó a Rimoldi, el Consejo de la Facultad, órgano presidido por el Decano, iniciaba un camino en paralelo en torno a la formación en psicología. El $1^{\circ}$ de abril de 1950, el Consejo resolvió convocar a los doctores Juan Carlos Brito del Pino y Mario Berta y "a la señorita Rebeca Millies" para elaborar un programa de Psicología Experimental a brindar en un curso durante 5 meses en FHC (Facultad de Humanidades y Ciencias, 1950). La resolución daba un plazo de 20 
días y el 19 de abril de 1950 la propuesta firmada únicamente por el Dr. Mario Berta fue enviada al Consejo de la FHC. En esa misiva, además de las características del curso se alertaba sobre la dificultad de llevar adelante el programa ante la carencia de un laboratorio y materiales adecuados, para lo que se propuso utilizar las instalaciones y materiales de los laboratorios de la Clínica Psiquiátrica, del Instituto de Fisiología, de Psicopedagogía Sebastián Morey Otero y el de Psicología de la Aeronáutica Militar (Berta, 1950).

La propuesta del Dr. Mario Berta se adelantaba a la solicitud que realizaría Jiménez de Aréchaga a Horacio Rimoldi unos meses después, evidenciando un juego de intereses que no se dirigía sobre un mismo objetivo. El 23 de febrero de 1951, el Consejo de FHC definió las bases de un llamado a concurso para el Curso de Psicología de la Licenciatura de Filosofía al que se presentaron Juan Carlos Brito del Pino, Mario Berta y Rebeca Millies. Las bases del concurso además de incluir la valoración de los méritos de cada postulante, preveía la realización del curso de psicología de manera paralela por cada postulante que sería seguido por estudiantes y sobre el cual se realizaría un informe al final del mismo. El tribunal debía visitar por lo menos 6 sesiones de trabajo de cada postulante durante los tres meses y se propuso que estuviese integrado por el Dr. Elio García Austt, el Dr. J. C. Montaner y el Dr. Horacio Rimoldi (Facultad de Humanidades y Ciencias, 1951a).

El Dr. Rimoldi, recientemente llegado al Uruguay, se vio rápidamente formando parte de un proceso de concurso para un cargo de un curso de Psicología que él no brindaría, sin claridad de la vinculación entre el mismo y el proyecto para el que había sido convocado por el Decano de la Facultad. En un repartido del Consejo de la FHC (1951b) se informó que el único postulante que se presentó a dar las clases fue el Dr. Mario Berta. Al mismo tiempo que se procesaba el concurso, Rimoldi iniciaba las actividades para las que había sido convocado, reuniéndose con 27 interesados en cursar psicología, a los que les realizó una entrevista breve con el fin de sondear los niveles de preparación, vocación e intereses especiales. Como resultado de las entrevistas Rimoldi definió dictar dos cursos, uno de carácter libre y otro limitado a solicitud de un grupo de estudiantes. El contenido del curso libre versó sobre: Historia de las pruebas mentales, Metodología y crítica de las pruebas mentales, Teorías modernas sobre inteligencias y su relación con las pruebas mentales, finalizando con el Estado actual de las investigación psicológica en los problemas relacionados con las teorías de la inteligencia y con la orientación y selección vocacional. El curso restringido trató sobre Estadística en 
Ciencias Sociales, especialmente en Psicología, y el objetivo fue que sirviera de base para cursos futuros (Rimoldi, 1951b).

En el mes de mayo, Horacio Rimoldi presentó el Memorándum para la creación del Instituto de Psicología y la organización de los estudios para la Licenciatura en Psicología, planteando que: “deberá encargarse en primer lugar de la investigación psicológica pura y en segundo lugar de la enseñanza de la psicología” (Rimoldi, 1951c, p.2). El Instituto contaría con un Director, profesores de tiempo completo, profesores asistentes, ayudantes de investigación, ayudantes de enseñanza y personal administrativo. Los profesores además de las actividades de investigación y enseñanza, podrían desempeñarse como consejeros de los estudiantes, actividad a la que le asignaba una fuerte responsabilidad en la formación más adecuada de los alumnos. Para la obtención del grado de Licenciado se proponía un mínimo de tres años de estudios y no más de seis y la culminación con una Tesis sobre un tema psicológico acordado con el profesor consejero, la continuidad de los estudios se preveía con la futura creación del Doctorado en Psicología.

El 21 de junio de 1951, en una carta dirigida a Louis Thurstone, Rimoldi le expresa que su actividad se ha centrado en la enseñanza debido a que la investigación se limitó por el escaso avance en la formación del Laboratorio de Psicología. Rimoldi le expresó a su maestro que en los últimos tres meses: "the main preocupation of the Faculty authorities has been a very disturbing affair, with political and other implications" (Rimoldi, 1951d). La compleja situación refería a la renuncia de Jimenez de Aréchaga y a las dificultades que tuvo la FHC en definir su dirección. Durante 1951 la FHC tuvo tres decanos: el Dr. Justino Jimenez de Aréchaga, el Dr. Fructuoso Pittaluga y el Dr. Emilio Oribe, esto complicó la organización de la institución y dejó a Rimoldi sin su principal apoyo. La falta de aliados, el laboratorio carente de materiales y aparatos, evidencian las dificultades que padecía, que también se reflejaban en su estado de salud. Rimoldi (1951d) le expresó a Thurstone que desde que llegó a Uruguay no se ha sentido muy bien: since I arrived here I have been suffering from a bad eczema....Moreover on account of the troubles in the School I have been quite worried and this has affected a bit my hearing. If things get finally well organized I might also feel physically better. Indeed, a psychosomatic case....

Las dolencias mencionadas por Rimoldi, seguramente estuvieron vinculadas al aislamiento y al tenso clima de competencia en el que se vio involucrado. En ese primer semestre de 1951, Mario Berta dictó el curso de Fundamentos Psicológicos del 
Psicoanálisis incluyendo investigación teórica y experimental. En un informe entregado a la FHC, Berta (1951) dejó asentado que: "desde mediados del mes de Abril se inició la tarea de Laboratorio en forma individual con cada estudiante" (p.14). Se planteó el objetivo de hacerles vivenciar a los estudiantes distintos procesos psíquicos como la proyección, a través de láminas del Thematic Apperception Test y la asociación espontánea con el Test de Asociación de Jung-Riklin. Rimoldi por su parte, luego de dos notas enviadas al Decano, el 20 y 29 de junio, expresando las necesidades del Laboratorio que intentaba crear, fue recibido el 1 de agosto por el Decano Pittaluga y logró que se respondiera parcialmente a algunos de sus pedidos. El 13 de septiembre envió al Consejo de FHC un informe sobre la actividad del Laboratorio reiterando la solicitud de materiales, el 29 de octubre informó sobre la cursada de los alumnos y al día siguiente, en una nueva nota informó sobre los cursos que dictaría en 1952. El 8 de noviembre nuevamente informó al Decano y al Consejo sobre sus ocho meses de actividad, haciendo un recuento pormenorizado de las carencias y dificultades con las que llevó adelante su tarea (Rimoldi, 1951a). Al final del año, el 30 de diciembre, Berta informó sobre el Curso de Psicología para estudiantes de la Licenciatura en Filosofía durante 1951. El informe se dividió en enseñanza de cátedra, laboratorio y seminario, detallando exhaustivamente horas trabajadas, tareas, contenidos temáticos abordados, métodos utilizados, juicios sobre algunos estudiantes y hasta una propuesta de cursada sumando otros cursos y la demanda de contar con colaboradores para llevar adelante tan exigente curso (Facultad de Humanidades y Ciencias, 1952). El año 1951 refleja un intenso trabajo de los diferentes actores por ubicar sus propuestas en el interés de la FHC y hacerse cargo de organizar la formación en psicología.

El año siguiente inició con la misma tónica, el Consejo de FHC en su sesión del 25 de enero, tiene en el orden del día el informe de la Comisión de Concursos referido al fallo del tribunal sobre el cargo de Psicología. El 22 de enero, el tribunal define que: "el único aspirante, Doctor Mario Berta, ha demostrado condiciones indiscutibles de capacidad docente; que ha revelado conocimiento amplio del tema elegido...que ha demostrado, asimismo, poseer vocación para el ejercicio de actividades de orden psicológico" (Facultad de Humanidades y Ciencias, 1952). El tribunal cambió su integración, no participaron el Dr. J. C. Montaner y tampoco el Dr. Horacio Rimoldi, quien durante enero se encontraba en Buenos Aires dictando un curso sobre Teoría de los Tests Mentales. La nueva integración contó con Carlos Vaz Ferreira, Emilio Oribe y García Austt, todos integrantes del Consejo de la FHC en ese momento. 
El ambiente en la FHC se presentaba cada vez menos propicio para los intereses de Rimoldi. En una nueva carta a Thurstone el 11 de febrero de 1952, le anunció que había iniciado contactos con amistades de los EUA para volver y asumir alguna plaza en ese país. A su vez, reafirmaba las dificultades con las que se encontró en Montevideo, mucho más complejas que lo acontecido en Mendoza (Rimoldi, 1952a). El 24 de marzo escribió nuevamente a Thurstone manifestando que la situación había empeorado, refiriéndose explícitamente a la resolución del concurso de psicología dos meses antes: "A man without training was appointed, during my absence, full Professor of Psychology, in spite of my opinion on the contrary. The fact is that he has good connections and political influence" (Rimoldi, 1952b). En la misma misiva Rimoldi afirmaba su decisión de abandonar Montevideo tan pronto como recibiera una buena propuesta de trabajo, reconociendo que "otherwise my scientific career may be seriously damaged" (Rimoldi, 1952b).

El primer semestre de 1952 contó con dos propuestas de cursada en psicología. Rimoldi propuso dictar el curso limitado de Teoría de Pruebas Mentales, curso avanzado de Psicometría y un curso libre de Introducción a la Psicología. Berta por su parte, propuso dictar un curso de Psicología de la Personalidad, anunciado como curso crítico teórico-experimental. El 2 de septiembre de ese año, Berta escribió al Decano Interino el Dr. Emilio Oribe informando sobre la situación de la Cátedra de Psicología de esta Facultad. En esa comunicación expresó que la enseñanza de la psicología debía contar con materiales y espacios para desarrollar las tareas experimentales, ya que existía un verdadero interés de los estudiantes por la psicología experimental y subraya que sería importante que se dicte un: "Curso crítico de Psicología experimental", donde se muestren "los pretendidos alcances de ciertos psicodiagnósticos y las exageraciones ilusorias de la cuantificación psicológica" (Berta, 1952a, p.1). Con este curso, Berta pretendía esclarecer cierta 'confusión reinante' en materia de experimentación psicológica y a la vez, insistía en la instalación de un laboratorio de psicología. Finalizaba reconociendo las dificultades económicas que hacían difícil instalar el laboratorio, sin embargo deslizaba una propuesta:

La Facultad de Humanidades y Ciencias dispondrá, dentro de corto tiempo, de un local y material técnico, creados y empleados hasta el momento para la Experimentación Psicológica, por el Dr. Horacio Rimoldi. Tanto el local como el material utilizado tienen carácter específicamente psicológico. Creemos que esta es la oportunidad menos onerosa 
para unificar y perfeccionar la Enseñanza de la Psicología en la Facultad. (Berta, 1952a, p.3)

Este gesto de unificación podría sugerir un acercamiento entre ambos profesores, pero un informe de Rimoldi nueve días después pone en duda esta suposición. El 11 de septiembre, Rimoldi reiteró las dificultades que encontró para realizar su tarea, y detalló cada una de las acciones que realizó para crear y hacer funcionar el laboratorio, nada menciona de una posible unificación. De todas maneras, el fin del camino de Rimoldi en Montevideo estaba cerca. Thurstone le escribe el 23 de septiembre para informarle que se verían próximamente, al momento en que Rimoldi asumiera su nuevo cargo en Princeton.

La partida se dio en el segundo semestre de 1952 y Berta quedó como único postulante a dirigir la formación en psicología de la FHC. En junio de 1953 Berta le hace llegar al Decano Vaz Ferreira un proyecto de presupuesto mínimo para el Laboratorio de Psicología. La propuesta incluyó la solicitud de una suma de dinero para compra de material psicológico, el nombramiento de un ayudante de clase y cuatro ayudantes de Laboratorio especializados y duplicar el rubro mensual del Laboratorio de 100 \$ a 200 \$ (Berta, 1953). La creación de una propuesta formativa en psicología quedaba así en manos del Dr. Mario Berta y del círculo de médicos alineado en torno a su accionar.

Una de las colaboradoras de Rimoldi durante los años que estuvo en Uruguay fue Elida Tuana (1913-2015), quien entrevistada años después dijo sobre esa situación que: “...los médicos no admitieron que un psicólogo y médico argentino, de gran predicamento, que se había formado en psicología con Thurstone, nada más y nada menos, se quedara aquí...” (Monetti y Ocampo, 2011). Juan Carlos Carrasco (1923-2010), quien compartió la formación con Mario Berta durante los primeros años y que luego fue un actor fundamental para la psicología autónoma, reafirmó las palabras de Tuana: "Sospecho y creo que el impedimento venía más por la Facultad de Medicina de Uruguay que por argentino (Rimoldi). Sin duda, gente de mucha influencia dentro de la facultad de medicina lo desplaza" (Oiberman, 2001).

\section{Rimoldi y el proyecto de psicología científica en Argentina y Puerto Rico}

En Argentina, las carreras de psicología planificadas por el Estado con perfil psicotécnico, se orientaron al ejercicio de la profesión liberal enfocada a la clínica 
psicoanalítica, e ideológicamente reacias a las estadísticas y a los laboratorios (Klappenbach, 2018).

Presidido por Bernardo Houssay, en 1958 se creó el Consejo Nacional de Investigaciones Científicas y Técnicas (CONICET), que fue inicialmente concebido con la misión de fomentar la investigación en las universidades. Desde valores universalistas buscando satisfacer estándares internacionales, se creó la carrera de investigador científico, complementando los salarios de selectos profesores para que se dediquen tiempo completo a esta actividad. Se implementaron políticas de subsidios a la investigación, contratación de investigadores extranjeros y becas al exterior para la formación de posgrado. Bajo el predominio de las ciencias biomédicas, y entre los debates sobre la supremacía de la investigación básica o aplicada al desarrollo socioeconómico del país, se abrieron paso las primeras becas y subsidios para el desarrollo de una ciencia psicológica, explícitamente alejada del modelo dominante en las universidades locales (Piñeda, 2020).

Con todo, en algunas universidades existían grupos de académicos que alentaban una formación alternativa. Para aquellos de la Universidad Nacional de Córdoba (UNC), la Universidad de Buenos Aires (UBA) y el CONICET cuya correspondencia analizamos, precisamente Rimoldi representaba una alianza oportuna para promoverla. Poco después de la experiencia uruguaya, el eminente académico procuró regresar a Latinoamérica. Ganó por concurso el cargo de Profesor de Psicología en la Facultad de Filosofía y Letras de la UBA, pero renunció a este sin asumir, permaneciendo en Chicago. Sin embargo, ese mismo año se abrieron nuevas posibilidades. Según relataba el Decano Tomás Fulgueiras en la carta dirigida a Rimoldi el 1 de febrero de 1957, la Facultad de Filosofía y Humanidades de la UNC, que estaba iniciando la carrera de psicología con grandes dificultades, lo invitó a dictar asignaturas de su predilección porque "se requiere de hombres de vocación, experiencia y conocimientos profundos para que esto no fracase o termine en una burda mistificación". Una serie de misivas intercambiadas entre Fulgueiras, Rimoldi y el Director del Instituto de Psicología Prof. Raúl Piérola, retratan la aceptación de Rimoldi, el agradecimiento de la Facultad y sus gestiones para contratarlo "en nombre del avance de la psicología argentina con la esperanza de que algún día se cuente entre el plantel estable" (Fulgueiras, 1957). Durante el segundo semestre de 1957 había aceptado ser Profesor de Psicotecnia y Orientación Profesional. En cambio, declinó a la sugerencia de Piérola de dictar Psicología Clínica por no ser materia de su especialidad, si bien reconocía que diseñaba experimentos sobre estos 
temas. La Facultad se comprometía a la compra de pruebas en Francia y en Estados Unidos, a pagar el traslado internacional y una modesta remuneración que podía incrementarse si aceptaba realizar tareas complementarias de orientación profesional o dictar cursos para Maestros Normales para el Ministerio de Educación de la Provincia, pero se le solicitaba al Profesor que tuviera a bien traer todos los libros especializados que pudiera cargar con él. El curso que diseñó Rimoldi, excedía la aplicación de pruebas psicotécnicas. Se orientó directamente a la formación para la investigación psicométrica. Planificó entre 50 y 60 clases para impartir nociones sobre pruebas paramétricas y no paramétricas y teoría de la medición en psicología. Estas preparaban para la realización de un trabajo final consistente en la tipificación de un grupo de pruebas mentales en población escolar secundaria de Córdoba, basada en experiencias previas que Rimoldi condujo en Mendoza y en Montevideo. Pese a las demoras para la compra del pasaje y de los pagos establecidos en el contrato, el curso se desarrolló según lo pautado. Esperaban repetir la experiencia al año siguiente para dictar Diseño de Experimentos, pero las "dificultades burocráticas" no lo facilitaron (Piérola, 1959). En 1959 se iniciaron gestiones mediante organismos extrauniversitarios -como la Comisión para el Intercambio Educativo entre Estados Unidos y Argentina a cargo del Dr. Jorge Tristán Bosch- que pudieran subsidiar la contratación de este y otros profesores visitantes (sobre los que se le pedía recomendaciones a Rimoldi). El contrato se gestionó para el segundo semestre de 1961 porque Rimoldi lo sujetaba a la condición de cumplir previamente con una invitación que le había realizado la Universidad de Puerto Rico. Sin embargo, las huelgas administrativas impidieron concretar a tiempo. Por lo que el Profesor de Loyola, lamentaba "el mal de fondo, sin esperanzas de pronto arreglo de las universidades argentinas" (Rimoldi, 1961). Viajó a Puerto Rico, después a Buenos Aires y regresó a Chicago. Desde su ciudad natal, en carta del 22 de agosto de 1961 le confiaba al colega portorriqueño Juan Nicolás Martínez que visitó "amigos en la Universidad local y, aunque se notan progresos, el ambiente universitario está demasiado politizado”. No obstante, en nuevos laboratorios y centros de estudio, quedó “impresionado al ver cómo la gente de aquí con escasos medios hace cosas verdaderamente increíbles".

Con todo, los cursos de 1957 en Córdoba fueron la oportunidad para que Rimoldi estableciera contacto con los estudiantes Enrique Saforcada, Hermelinda Fogliatto y Rosalía Amitrano de Paiva que se convirtieron en sus discípulos, y con el Centro de Investigaciones Acústicas y Luminotécnicas (CIAL) dirigido por el Ingeniero Guillermo Fuchs en la Facultad de Arquitectura y Urbanismo, a quienes siguió asesorando en 
investigaciones interdisciplinarias en colaboración con la carrera de psicología. Esto motivó en Rimoldi la iniciativa de gestionar un convenio entre la Universidad de Loyola y CONICET para promover estancias de posgrado de estudiantes de universidades argentinas. El convenio fue firmado. Al laboratorio de Rimoldi se encaminaron Fogliatto y, años después, Paiva para realizar su doctorado. Saforcada, quien también fue aceptado, no lo pudo concretar. Se inclinó por una especialización local en sociología para la que se contrataron varios profesores estadounidenses. Asimismo, Rimoldi no perdía ocasión de agenciar los favores de CONICET para el fortalecimiento del Instituto de Psicología de Córdoba, por medio de su Presidente Bernardo Houssay y el miembro del Directorio Luis Federico Leloir (1906-1987).

Mientras tanto, la invitación a la Universidad de Puerto Rico (UPR) había sido propiciada por Mercedes Rodrigo. Ella se había radicado en el país con su hermana María, tras circunstancias políticas que habían dificultado su vida en Colombia (Ardila, 1999). En carta del 22 de agosto de 1961, se dirigía afectuosamente a las hermanas. Les confiaba sus temores sobre la difícil situación política y económica que percibía en su país natal, siendo este un gran obstáculo para su regreso definitivo y para el desarrollo de la psicología científica.

Mercedes Rodrigo trabajaba en el Hospital de Veteranos, institución que jugó un rol fundamental en los inicios de la psicología clínica portorriqueña. Contaba con una Unidad Psiquiátrica donde se realizaban evaluaciones psicológicas a cargo de un nutrido equipo profesional, frecuentemente formado en Estados Unidos con la asistencia financiera de la Oficina de Veteranos de ese país (Bernal, 2013). Por otra parte, la psicóloga española también presidía la Sociedad de Psicología de Puerto Rico que conjuntamente con la Universidad invitaba a Rimoldi a dictar el curso de Teoría del Diseño Experimental del 15 de enero al 15 de febrero de 1959 (Rodrigo, 1958). Este tipo de acuerdos entre la asociación y la universidad era habitual en la gestión de profesores visitantes, frecuentemente de Estados Unidos, como el Dr. Robert Ladd Thorndike de la Universidad de Columbia (1910-1990) o Starke Hathaway (1903-1984) de la Universidad de Minnesota y constructor del MMPI. La colaboración entre las dos organizaciones también había logrado en el país la celebración del IV Congreso Interamericano de Psicología en 1956, presidido por Otto Klineberg. Para esa época muchos psicólogos habían cursado sus doctorados en Estados Unidos, se desempeñaban allí como Profesores Visitantes, y se mantenían vínculos fluidos con la American Psychological Association y otras asociaciones estatales de psicología estadounidenses (Roca de Torres, 2006). En el 
marco de un proceso de industrialización, y aún con las resonancias de la conferencia de Boulder para el entrenamiento en psicología, se asistía al desarrollo de la psicología clínica y de la psicometría. El Departamento de Investigación Pedagógica y Estadística de Instrucción Pública se interesaba por producir adaptaciones como la prueba de inteligencia de Weschler para niños. También se tradujo Binet-Stanford y normalizaciones de las pruebas de Figura Humana, Rorschach y Apercepción Temática, entre otras (Bernal, 2013). En ese contexto, Rimoldi fue muy bien recibido por los miembros de la Asociación, y de diversas dependencias universitarias que representaban campos disciplinares y profesionales muy disímiles: la Escuela de Educación, el Departamento de Psicología, el Colegio de Ciencias Sociales, el Hospital de Psiquiatría y el Colegio de Administración de Negocios. Referentes de todos ellos le escribieron expresando su aprecio y deseo de mantenerse en contacto. Así, el Director del Hospital de Psiquiatría consideraba que Rimoldi supo dejar "una muy buena estela" que "habrá de ayudar mucho al desarrollo de la psicología de Puerto Rico" (Roselló, 1959). Nuevos intercambios se produjeron en consecuencia.

Una de las estudiantes del curso, Ada Izcoa, continuó con su formación doctoral en Loyola bajo la dirección de Rimoldi. Contribuyó a la línea de investigación sobre procesos de resolución de problemas desde los estudios del pensamiento esquizofrénico. Así, se convirtió en la primera mujer doctorada en psicología de Puerto Rico (Bernal, 2013). Entre los agradecimientos que pronunciaba en su tesis, incluía a H. Fogliatto (Izcoa, 1965).

Más allá de la frecuente circulación de doctorandos a Estados Unidos, la UPR proyectaba crear su Escuela de Graduados. Contaban con un anteproyecto asesorado por académicos de Pennsylvania y aprovechaban la cercanía de Rimoldi para pedirle también su opinión. El proyecto involucró a las Facultades de Ciencias Sociales, Pedagogía y Comercio, como se deja saber en las cartas que entre el 21 y el 22 de agosto de 1961 Rimoldi dirigió por separado al Dr. Álvarez, Decano de la Escuela de Comercio, a la Dra. Adriana Guzmán, Decana del Colegio de Ciencias Sociales, al Jefe del Departamento de Psicología Juan Nicolás Martínez, al Director del Instituto de Estadísticas del Colegio de Administración de Negocios, Michael Cooney y a la misma Mercedes Rodrigo. Más allá de las variaciones de cada carta, en todas se leía idéntico párrafo manifestando su discrepancia y especial preocupación:

No discute algunos puntos fundamentales que atañen a lo que debe ser una verdadera Escuela de Graduados e ignora, en gran medida las características y 
posibilidades de Puerto Rico [...] Un asunto tan serio para Puerto Rico y para todos los países de habla hispana en donde no hay verdaderas Escuelas de Graduados, se debía proceder con máxima atención.

En el mensaje a la Decana de Ciencias Sociales también se aprecia que ella había tomado cursos con Rimoldi y que le había pedido al Profesor asesoramiento para contratar docentes de ciencias básicas para el Departamento de Psicología.

Con Michael Cooney intercambió una larga serie epistolar. En su misiva del 6 de febrero este lo invitó a dictar un curso de Estadística Aplicada, dirigida a académicos, graduados y unos pocos estudiantes de grado avanzados de psicología, sociología y educación que integraban proyectos de investigación del Instituto, entre los meses de junio y julio de ese año. El 20 de febrero Rimoldi aceptó. Recomendaba bibliografía de apoyo sobre diseño avanzado de experimentos. Un aula colmada de asistentes con entrenamiento en la materia lo esperaba con altas expectativas. Los mensajes denotan el afecto entre Rimoldi y la familia de Cooney y la presencia de Mercedes Rodrigo en la organización de su recepción y alojamiento.

Finalmente, el Jefe de la Sección Orientación Vocacional, Entrenamiento y Ajuste de la Oficina Regional de Veteranos R.G.Cundiff, en su carta del 19 de enero de 1961, agradeció a Rimoldi por el tiempo que él y Ada Izcoa le habían dedicado, discutiendo los problemas de evaluación en Puerto Rico. También le expresaba su gratitud por el material bibliográfico que le proporcionó y por la disposición para dialogar con representantes de la Oficina Central en Washington o de la Regional de Chicago, que ya estaban informadas.

La correspondencia con Puerto Rico cesó en 1965. Mientras tanto, desde 1961 otro grupo de misivas reflejan un creciente proceso de colaboración de Rimoldi con académicos de la Facultad de Medicina de la UBA y de CONICET que culminaría en 1970 con su radicación definitiva en Buenos Aires, donde se aspiraba a organizar un polo científico dirigido por él.

Jorge Insúa, Titular de la Cátedra de Psicología Médica de la UBA, le pedía asesoramiento en el Programa de estudios de la Residencia en Psicología Médica y Psicología Clínica que la Facultad de Medicina le encomendó organizar en el Hospital de Clínicas y también lo invitaba a dictar un seminario para "imprimir la correcta orientación”. Insúa abrigaba la esperanza de remediar por la vía del posgrado, lo que juzgaba una incorrecta formación en las carreras de grado en psicología. Contaba con que "comiencen a llegar algunos de los formados en el extranjero y que otros de la cátedra 
vayan saliendo del país para adquirir formación que no les dan en nuestras facultades de psicología". En el objetivo del Programa, Insúa reflejaba su concepción del rol profesional del psicólogo en el campo clínico, limitado a tareas de investigación, que “conozca ampliamente las técnicas de su especialidad, con iniciativa personal y espíritu creador, eficaz colaborador del médico en la difícil tarea del diagnóstico y pronóstico de las afecciones mentales y que pueda integrarse en el constructivo trabajo en equipo" (Insúa, 1967). Rimoldi no coincidía en esa visión. La juzgaba superada en Estados Unidos y en países europeos donde los psicólogos son autónomos en el ejercicio de la psicoterapia, campo en el que logran formarse mejor que los psiquiatras, de acuerdo a su respuesta del 28 de diciembre de 1967, en la que aconsejaba ampliar los horizontes:

What I am very much afraid of is that if you are in a way starting medical psychology in Argentina, you should not do it in such a way that the limitations that you introduce from the very beginning will hamper the further development of clinical psychology in our country. [...] One has to set certain aims [...] to change what we do not consider to be right in the present situation.

Las cartas con invitaciones a la Argentina se volvieron más frecuentes en esos años. En 1965 se organizó una gira de Rimoldi por Buenos Aires, Córdoba, San Luis y Mendoza para dictar 15 conferencias, entre las que se incluía su asistencia a la $1^{\circ}$ Jornada Argentina de Orientación Vocacional presidida por Nuria Cortada, y al $2^{\circ}$ Congreso Argentino de Psicología, por Plácido Horas. Esta gira fue subsidiada por CONICET porque, al decir de Cortada en carta a Rimoldi del 18 de agosto de 1965, las universidades no contaban con fondos suficientes. El apoyo de CONICET a las actividades formativas que incluían a Rimoldi también se expresó en el otorgamiento de Becas a graduados del entorno de Medicina cercano a Houssay para estudiar en el Laboratorio de Psicometría de la Universidad de Loyola. Entre ellos, el Dr. Pecchi Saavedra del Instituto de Medicina y Biología Experimental, quien logró una estancia rentada para investigar mientras contribuía enseñando psicofisiología. Ana María Insúa, hermana del mencionado catedrático de Psicología Médica obtuvo beca para estudiar estadística e iniciar el doctorado bajo la dirección de Rimoldi, que culminó en 1970. También fue becada la Dra. Ives Lys Danna, Profesora en Filosofía y Ciencias de la Educación por la Universidad Nacional de La Plata (UNLP) y doctorada en Psicología en La Sorbona, donde estudió con Jean Piaget (1896-1980) y René Zazzo (1910-1995). Tomó un curso de posgrado que Rimoldi dictó en UNLP. Para el 9 de octubre de 1962, cuando le solicitó su admisión en 
Loyola ya era Investigadora de CONICET y se desempeñaba en el Hospital de Clínicas bajo la dirección de Telma Reca (1904-1979).

Las cartas de esos años también documentan un papel activo de Rimoldi en las evaluaciones y asesoramientos que la comisión de Psicología de CONICET le solicitó. En una oportunidad requerían referencias de expertos en "Psicología Experimental" para integrar dicha comisión. Él recomendó a sus discípulas Nuria Cortada y Hermelinda Fogliatto (Rimoldi, 1968).

Rimoldi no perdía las esperanzas de regresar a su país natal, en el que permanecía su familia. Los referidos compatriotas (Cortada, 1966, Danna, 1967, Insúa, 1968) creían fervientemente que el futuro de la ciencia psicológica en Argentina dependía de que le brindaran las condiciones necesarias para organizar un instituto de investigaciones local bajo su dirección, y abogaban por esta causa ante Houssay y los demás miembros del Directorio de CONICET. Finalmente, esa meta fue alcanzada en 1970 con la creación del CIIPME.

\section{Discusión}

El conjunto documental analizado reconstruye abundantes datos empíricos que aportan al avance de las historias locales desde una perspectiva policéntrica. En particular, se ha procurado visibilizar la circulación de conocimientos y de prácticas formativas, con sus asimilaciones y resistencias, en el marco de procesos de profesionalización de la psicología. Se verifica que, cuando Rimoldi se desempeñaba exitosamente en Estados Unidos, colaboró denodadamente con el fortalecimiento de centros de investigación para el desarrollo de una psicología científica en países latinoamericanos. El modelo que auspició logró el alcance que permitió cada contexto. Más allá de las adversidades políticas y presupuestarias de las universidades de Uruguay y de Argentina que impedían estructuralmente la formación en psicología centrada en la investigación, las resistencias allí generalmente obedecían a las disputas con el campo médico por el control de la carrera de psicología y por la hegemonía en el ejercicio profesional de la psicoterapia. Pese a ser médico, precisamente en un momento donde la psicología pugnaba por lograr autonomía frente a la medicina, acaso la internacionalización de la trayectoria de Rimoldi contribuyó a despegarlo de la visión subordinada de la psicología, alentando nuevos horizontes. Asimismo, es de notar que en Argentina, las políticas científicas eran controladas por académicos del campo biomédico que validaban prácticas y distribuían recursos, por lo que en ese territorio la psicología debió ajustarse a sus parámetros. De 
esa manera, se encontró un intersticio para el acceso temprano de psicólogos al sistema científico cuando la profesión de investigador estaba en sus albores. Con condiciones muy disímiles, la permeabilidad de Puerto Rico a la psicología de Estados Unidos fomentaba la especial valoración de las oportunidades que Rimoldi representaba para académicos de diversas procedencias disciplinares y, sin embargo, centrados en la investigación científica en psicología. Seguramente, a ello también habrá contribuido la amistad con Mercedes Rodrigo, quien ocupaba un lugar significativo en ese escenario.

Para finalizar, como advirtió Danziger (1996), las historias policéntricas permiten analizar aspectos del conocimiento psicológico en el marco de particulares relaciones de poder y entornos culturales, pero también deben dar cuenta de su complejidad. En este sentido, y reconociendo los límites de nuestro estudio, creemos necesario continuar con trabajos que permitan conocer el modo en que diferentes teorías y prácticas se trasmitieron y recepcionaron en diferentes países, generando comunidades académicas y profesionales locales. 


\section{Referencias}

Abbott, A. (1988). The system of professions: an essay on the division of expert labor. Chicago: The University of Chicago Press, 1988.

Ardila, R. (1999). Mercedes Rodrigo (1891-1982). Revista Latinoamericana de Psicología, 20(3), 429-434. Disponible en: http://www.uptc.edu.co/export/sites/default/eventos/2015/cf/ccp/doc/prim_psico log.pdf

Asociación de Psicólogos Universitarios Egresados de la Escuela de Tecnología Médica (1983). (Fort- da: Publicación de la Asociación de Psicólogos Universitarios Egresados de la Escuela de Tecnología Médica). Montevideo, 1983.

Benjamin, L. T. Jr (2006). A History of Psychology in Letters. Oxford: Blackwell Publishing.

Bernal, G. (2013). 60 Years of Clinical Psychology in Puerto Rico. Interamerican Journal of Psychology, 47(2), 211-226. Disponible en: https://www.redalyc.org/pdf/2332/233222863019.pdf

Berta, M. (1950, 19 de abril). [Comunicación al Decano Justino Jiménez de Aréchaga. Programa para un curso de Psicología Experimental]. Archivo Historia de la Medicina (Archivo Mario Berta. Archivo sin catalogar. Facultad de MedicinaUdelaR).

Berta, M. (1951a, junio). [Informe de trabajo realizado durante el curso sobre Fundamentos Psicológicos del Psicoanálisis. (investigación teórica y experimental. Abril, mayo, junio 1951]. Archivo Historia de la Medicina (Archivo Mario Berta. Archivo sin catalogar. Facultad de Medicina- UdelaR).

Berta, M. (1952a, 2 de septiembre). [Comunicación al Decano Dr. Emilio Oribe]. Archivo Historia de la Medicina (Archivo Mario Berta. Archivo sin catalogar. Facultad de Medicina- UdelaR).

Berta, M. (1953, 11 de junio). [Carta al Decano C. Vaz Ferreira. Proyecto de presupuesto para el Laboratorio de Psicología]. Archivo Historia de la Medicina (Archivo Mario Berta. Archivo sin catalogar. Facultad de Medicina- UdelaR).

Brock, A. (2014). What is a polycentric History of Psychology? Estudos e Pesquisas em Psicologia, 14(2), 646-659. Disponible en: http://www.epublicacoes.uerj.br/index.php/revispsi/article/view/12565/9749

Calabresi, C. V. (2010). Consideraciones epistemológicas en las Publicaciones del Instituto de Psicología Experimental de la Universidad Nacional de Cuyo (19431947). Fundamentos en humanidades, 11(21), 37-52. Sitio web: http://fundamentos.unsl.edu.ar/pdf/revista-21.pdf

Carbonell de Grompone, M. (Dir. Resp) (1947). Boletín del Laboratorio de Psicopedagogía "Sebastián Morey Otero" de los Institutos Normales, (3 y 4). Montevideo: Imprenta Nacional.

Carpintero, H. (2006). Horacio J. A. Rimoldi (1913-2006) y la psicología iberoamericana. Revista de Historia de la Psicología, 27(2/3), 225-232. Disponible en: https://www.revistahistoriapsicologia.es/archivo-all-issues/2006-vol-27

Cortada, N. (1966, 24 de noviembre). [Carta a Rimoldi]. CIIPME (Legado Rimoldi en Biblioteca del CIIPME. Archivo sin catalogar. Carpeta C).

Danna, Y. L. (1967, 18 de mayo). [Carta a Rimoldi]. CIIPME (Legado Rimoldi en Biblioteca del CIIPME. Archivo sin catalogar. Carpeta CONICET).

Chavez, J., \& Martinez Sena, P. (2021). Controversias sobre el ejercicio profesional de la psicología en Uruguay. Fractal: Revista de Psicologia, 33(2), 151-161. https://doi.org/10.22409/1984-0292/v33i2/49362 
Danziger, K. (1996). Towards a polycentric history of psychology. Paper presented at the 26th International Congress of Psychology in Montréal, Canada. Available at http://www.kurtdanziger.com/Paper\%209.pdf

Facultad de Humanidades y Ciencias. (1950, 1 de abril). [Expediente 275/850]. Archivo Historia de la Medicina (Archivo Mario Berta. Archivo sin catalogar. Facultad de Medicina- UdelaR).

Facultad de Humanidades y Ciencias. (1951a, 23 de febrero). [Bases para el concurso de Psicología aprobadas por el Consejo de la Facultad de Humanidades y Ciencias]. Archivo Historia de la Medicina (Archivo Mario Berta. Archivo sin catalogar. Facultad de Medicina- UdelaR).

Facultad de Humanidades y Ciencias. (1951b, s/d). [Repartido N. . 229. Información sucinta sobre la situación de los concursos en la Facultad]. Archivo Historia de la Medicina (Archivo Mario Berta. Archivo sin catalogar. Facultad de MedicinaUdelaR).

Facultad de Humanidades y Ciencias. (1952a, s/d). Repartido $N^{\circ}$ 40/952. Informe del Dr. Mario Berta sobre el desarrollo del curso de Psicología, para los estudiantes de la Licenciatura de Filosofía durante el año 1951. Archivo Historia de la Medicina (Archivo Mario Berta. Archivo sin catalogar. Facultad de Medicina- UdelaR).

Facultad de Humanidades y Ciencias. (1952b, 24 de enero). Orden del día Consejo de Facultad de Humanidades y Ciencias. Archivo Historia de la Medicina (Archivo Mario Berta. Archivo sin catalogar. Facultad de Medicina- UdelaR).

Freidson, E. (1996). Para uma análise comparada das profissões: a institucionalizaçao do discurso e do conhecimiento formais. Revista Brasilera de Ciências Sociais, $11(31), 141-154$.

Fulgueiras, T. (1957, 15 de febrero). [Carta a Rimoldi]. CIIPME (Legado Rimoldi en Biblioteca del CIIPME. Archivo sin catalogar. Carpeta Córdoba).

Gallegos, M. (2010). La primera Conferencia Latinoamericana sobre Entrenamiento en Psicología (1974): el modelo Latinoamericano y su significación histórica. Psicologia. Ciência e Profissão [online], 30(4), 792-809. DOI https://doi.org/10.1590/S1414-98932010000400010

Hereford, C. (1966). Current status of psychology in Latin América. Latin American Research Review, 1(2),97-108.

Insúa, J. (1967, 20 de noviembre). [Carta a Rimoldi]. CIIPME (Legado Rimoldi en Biblioteca del CIIPME. Archivo sin catalogar. Carpeta I.).

Insúa, J. (1968, 8 de abril). [Carta a Rimoldi]. CIIPME (Legado Rimoldi en Biblioteca del CIIPME. Archivo sin catalogar. Carpeta I.).

Izcoa, A. (1965). A Study of Schizophrenic Thinking in Problem Solving Tasks. Dissertations. 770. https://ecommons.luc.edu/luc_diss/770

Jacó-Vilela, A., Espírito-Santo, A., Degani-Carneiro, F., Goes, L. \& Vasconcellos, M. (2016). Investigando em História da Psicologia: contribuições metodológicas. Interacciones, 2(2), 123-134. DOI: https://doi.org/10.24016/2016.v2n2.39

Klappenbach, H. (2012). Informes sobre formación universitaria en psicología en Argentina. 1961-1975. Revista Interamericana de Psicología, 46(1), 181-192. Disponible en: https://www.redalyc.org/articulo.oa?id=28424858017

Klappenbach, H. (2014). Acerca de la metodología de la investigación en historia de la psicología. Psykhé, 23(1), 1-12. DOI http://dx.doi.org/10.7764/psykhe.23.1.584

Klappenbach, H. (2018). Cambios en los primeros perfiles de formación en el campo de la Psicología Argentina. Desde la Planificación Estatal a una Profesión Liberal. Revista_de Historia de la Psicología, 39(3), 18-27. DOI https://doi. org.10.5093/rhp2018a13 
Klappenbach, H., Vázquez-Ferrero, S., \& Gallegos, M. (2018). The long road to the profession of Psychologist. In R. Ardila (Ed.), Psychology in Latin America. Current Status, Challenges and Perspectives (pp. 27-51). Cham, Switzerland: Springer.

Monetti, D.; Ocampo, E. (2011). (Elida. J. Tuana. Yo y mis circunstancias. DVD). Montevideo: Mediateca- Facultad de Psicología- UdelaR.

Oiberman, A. (2001). (Pioneros de la psicología en Argentina. Historias de vida...cuando la vida es historia. Entrevista realizada a la Prof. Elida Tuana y al Prof. Juan Carlos Carrasco). Disponible en: https://dspace.palermo.edu/ojs/index.php/psicodebate/article/view/501/296

Oiberman, A. (2003). (Pioneros de la psicología en Argentina. Historias de vida...cuando la vida es historia Dr. Horacio J. A. Rimoldi). Disponible en: https://dspace.palermo.edu/ojs/index.php/psicodebate/article/view/500/295

Ortiz-Torres, B. (2013). Estado actual y posibilidades futuras de la psicología en cuatro países del Caribe: Puerto Rico, Cuba, República Dominicana y Haití. Revista Interamericana de Psicología, 47(2), 239-244. Disponible en: https://journal.sipsych.org/index.php/IJP/article/view/218

Piérola, R. (1959, 13 de marzo). [Carta a Rimoldi]. CIIPME (Legado Rimoldi en Biblioteca del CIIPME. Archivo sin catalogar. Carpeta Córdoba).

Piñeda, M. A. (2020). Bernardo Houssay, Horacio Rimoldi y la formación de investigadores en Psicología. Una bisagra entre CONICET y las Universidades Argentinas. Revista de Historia de la Psicología, 41(3), 2-15. Doi: 10.5093/rhp2020a10.

Revista Latinoamericana de Psicología (1986). Horacio J. A. Rimoldi. Revista Latinoamericana de Psicología, 18(3), 477-480. Disponible en: https://www.redalyc.org/pdf/805/80518309.pdf

Rimoldi, H.. J. A. (1951a, 8 de noviembre). [ Comunicación al Consejo de Facultad de Humanidades y Ciencias]. Archivo Historia de la Medicina (Archivo Mario Berta. Archivo sin catalogar. Facultad de Medicina- UdelaR).

Rimoldi, H.. J. A. (1951b, 5 de mayo). Comunicación al Decano de Facultad de Humanidades y Ciencias, Dr. Justino Jimenez de Arechaga. Archivo Historia de la Medicina (Archivo Mario Berta. Archivo sin catalogar. Facultad de MedicinaUdelaR).

Rimoldi, H.. J. A. (1951c, mayo). [Memorándum para la creación del Instituto de Psicología y la organización de los estudios para la Licenciatura en Psicología]. Archivo Historia de la Medicina (Archivo Mario Berta. Archivo sin catalogar. Facultad de Medicina- UdelaR).

Rimoldi, H.. J. A. (1951d, 21 de junio). [Carta a L. L. Thurstone]. CIIPME (Legado Rimoldi en Biblioteca del CIIPME. Archivo sin catalogar. Carpeta T).

Rimoldi, H.. J. A. (1952a, 11 de febrero). [Carta a L. L. Thurstone]. CIIPME (Legado Rimoldi en Biblioteca del CIIPME. Archivo sin catalogar. Carpeta T).

Rimoldi, H.. J. A. (1952b, 20 de marzo). [Carta a L. L. Thurstone]. CIIPME (Legado Rimoldi en Biblioteca del CIIPME. Archivo sin catalogar. Carpeta T).

Rimoldi, H.. J. A. (1961, 20 de abril). [Carta a Piérola]. CIIPME (Legado Rimoldi en Biblioteca del CIIPME. Archivo sin catalogar. Carpeta Córdoba).

Rimoldi, H.. J. A. (1968, 3 de octubre). [Carta a Dionisio Cámola]. CIIPME (Legado Rimoldi en Biblioteca del CIIPME. Archivo sin catalogar. Carpeta CONICET).

Rivera, B., \& Maldonado, L. (2000). Revisión histórica de la reglamentación de la psicología en Puerto Rico: 1954 a 1990. Revista Interamericana de Psicología, $34(2), 127-162$. 
Roca de Torres, I. (1999). La psicología en Puerto Rico. In A. Modesto \& Eagly, A. (Eds.), Psicología en las Américas (pp. 241-254). Caracas: Sociedad Interamericana de Psicología.

Roca de Torres, Irma (2006). La Asociación de Psicología de Puerto Rico: una perspectiva histórica (1954-2005). Revista Puertorriqueña de Psicología, 17, 241-276. Disponible en: https://www.redalyc.org/articulo.oa?id=233222863015

Rodrigo, M. (1958, 15 de diciembre). [Carta a Rimoldi]. CIIPME (Legado Rimoldi en Biblioteca del CIIPME. Archivo sin catalogar. Carpeta Puerto Rico).

Roselló, J. A. (1959, 8 de mayo). [Carta a Rimoldi]. CIIPME (Legado Rimoldi en Biblioteca del CIIPME. Archivo sin catalogar. Carpeta Puerto Rico).

Vessuri, M. C. (1994) La ciencia académica en América Latina en el siglo XX. Redes: Revista de estudios sociales de la ciencia, 1(2), 41-76. Disponible en http://ridaa.unq.edu.ar/handle/20.500.11807/304 